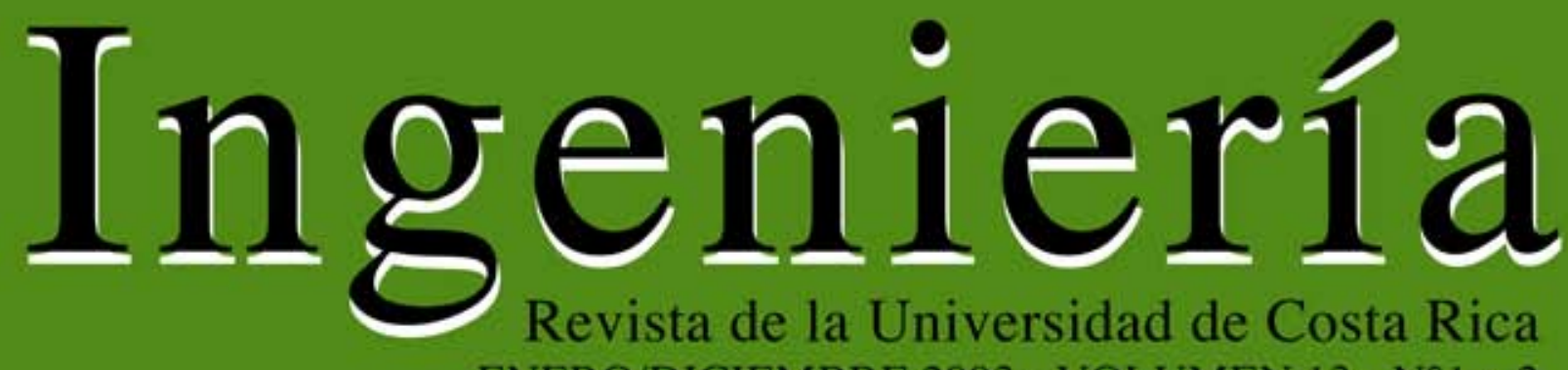
ENERO/DICIEMBRE 2003 - VOLUMEN 13 - N¹ y 2

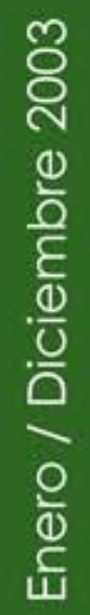

$\frac{m}{5}$
$\frac{c}{d}$
$\frac{5}{5}$
$\frac{\partial}{\circ}$

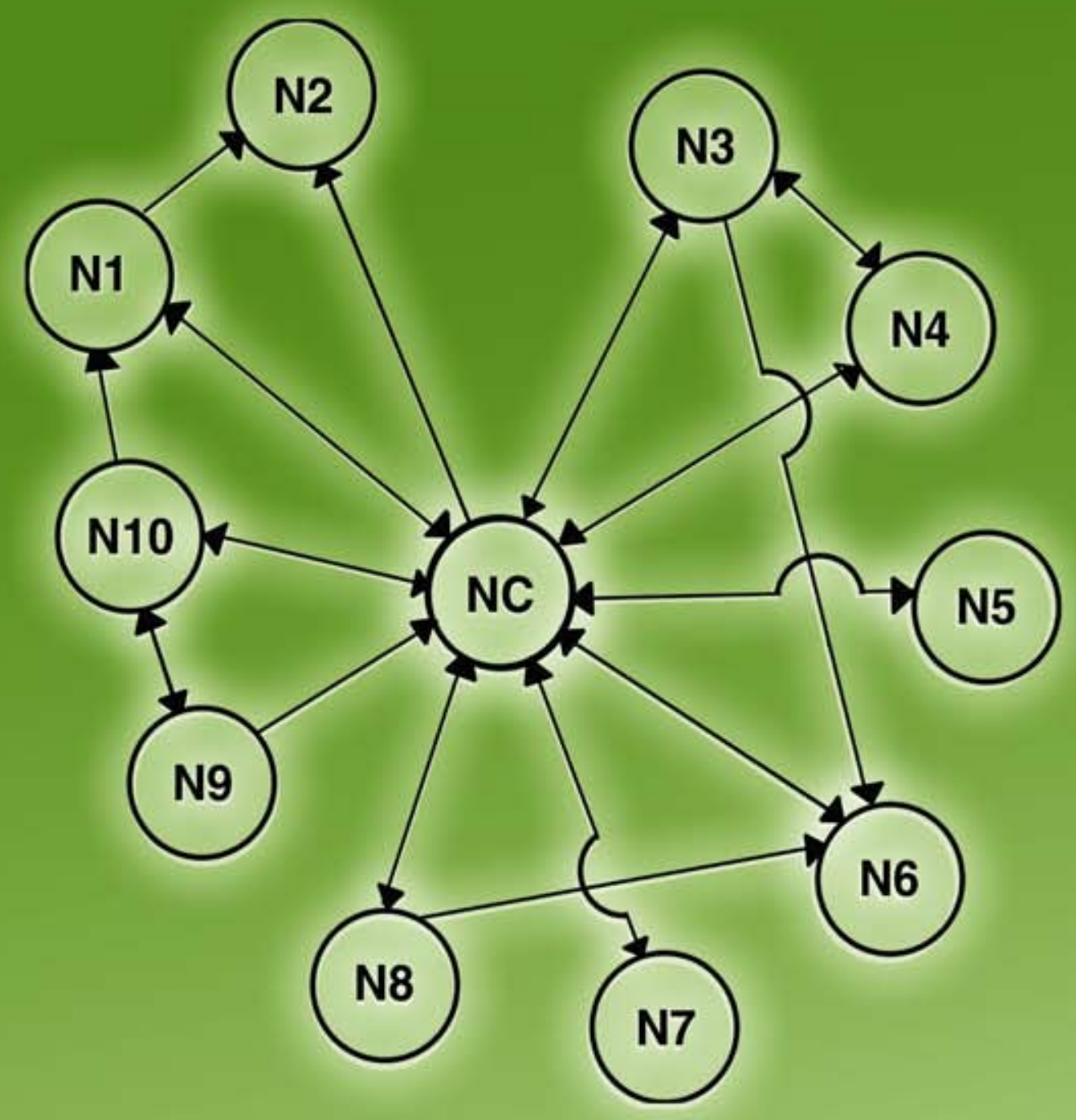

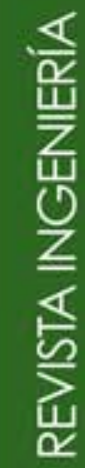




\title{
SOFTWARE EDUCATIVO: APORTE DE LAS UNIVERSIDADES ESTATALES COSTARRICENSES DURANTE EL PERÍODO 1990-2001.
}

Carlos Vargas Castillo

\begin{abstract}
Resumen
Se realiza un estudio sobre el desarrollo de software con fines educativos llevado a cabo en la Universidad de Costa Rica, el Instituto Tecnológico de Costa Rica, la Universidad Nacional y la Universidad Estatal a Distancia, durante el período comprendido entre 1990 y 2001, con el fin de conocer de manera integral el tipo de aporte brindado. Primeramente se analiza cuál ha sido la tendencia en producción de software educativo durante el período de estudio. Luego se determina cómo se distribuyen los estudios por universidad estatal y se identifican las unidades académicas y de investigación que han contribuido en dichos estudios, dentro de cada una de las universidades. Finalmente, se categorizan las áreas temáticas tratadas en las investigaciones, identificando aquellas que han sido objeto de mayor atención, así como las que han sido poco consideradas, o del todo desatendidas.
\end{abstract}

Palabras clave: software educativo

\begin{abstract}
A research on educational software, as developed by the Costa Rican State Universities between 1990-2001, is undertaken in order to assess their global contribution to the educational formal system. First, the educational software tendency, accross the twelve year time period, is displayed and analized. Next, a distribution of educational software estudies per university is show, and, organizational units suporting software development in the universities are identified, too. Last, major themes and subthemes, addressed by educational software developers at the universities over one decade of research, are identified.
\end{abstract}

Key words: educational software

\section{INTRODUCCIÓN}

El objeto de estudio de esta investigación es el software educativo desarrollado en las universidades estatales. Se utiliza el método de análisis de contenido documental y temático. Bermúdez (1982) afirma que "Sea cual fuere el tipo de análisis de contenido escogido, existen dos procedimientos básicos: el exploratorio o descriptivo y el verificativo de hipótesis". En esta investigación se adopta el primer procedimiento.

Pochet (2000) propone que la codificación del texto se efectúe mediante la segmentación del documento en unidades menores de las cuales se extraen los temas fundamentales, con base en palabras claves, y además que se establezca una diferencia entre los temas principales y los secundarios.

La variables de interés que se codificaron para este estudio son: el área temática abordada, el tópico o subtema tratado dentro del área temática, y la población meta a la que se orienta el software educativo. También se incluyen el año en que se publica la investigación y la identificación de la misma.

Las investigaciones sobre desarrollo de software con fines educativos son importantes para la Educación Costarricense, por cuanto, según Cabero (1999), permiten incorporar la tecnología 
informática al quehacer educativo con el fin de lograr cambios cualitativos en la educación.

De acuerdo con Galvis (1994), el rasgo distintivo de una aplicación educativa respecto a una meramente informativa, es que la primera se halla inmersa en un diseño concreto, o plan, determinado para enseñar algo a alguien (1994). Incorporar dicho rasgo en el diseño incrementa la complejidad del producto.

La complejidad de la producción de software educativo estriba en el hecho de que deben efectuarse decisiones en torno a los contenidos (selección, organización, adaptación a los usuarios, etc.); a las estrategias de enseñanza de dichos contenidos y a la forma de presentación (diseño de pantallas, interacción, tipos de navegación, etc.) más adecuadas con el objeto de facilitar el proceso de aprendizaje (Heller, 1991; Gros y Spector, 1994).

Una aplicación educativa debe mostrar intencionalidad y sistematización (Bauza, 1997). Por lo tanto, en el desarrollo de una aplicación orientada a la educación se debe considerar, además de un discurso y un mensaje, el añadir una estrategia de formación, la cual permitirá que ésta efectivamente sirva para enseñar.

\section{RECOPILACIÓN DE LOS DATOS.}

La fuente de datos utilizada en este estudio consistió de una serie de investigaciones sobre desarrollo y uso de software educativo realizadas por las universidades estatales. Dichas investigaciones fueron identificadas mediante búsqueda exhaustiva por palabras clave, con ayuda de sistemas de búsqueda computarizados y también manualmente. Algunas de las palabras clave compuestas utilizadas fueron: Software Educativo, Informática Educativa, Pedagogía y Computadoras, Computadoras y Aprendizaje.

No todos los trabajos, identificados mediante los procesos de búsqueda por palabras clave, resultaron pertinentes para los fines de este estudio. Luego de un breve escrutinio de los contenidos, se constató que, a pesar de que los títulos aludían al sujeto de estudio, los contenidos no enfocaban el tópico en concordancia con los requerimientos de la investigación.

Entre los documentos identificados, se incluyeron como fuente documental para este estudio, todas aquellas investigaciones en las cuales se elaboró algún producto de software concreto, con fines educativos, y en las que se estudió el fenómeno del desarrollo de software educativo, enfocándose en aspectos específicos respecto a modelos, métodos, diseño, o guías didácticas.

En total se analizaron cuarenta y ocho investigaciones que tratan el desarrollo de software educativo, realizadas en las universidades estatales de Costa Rica, durante el período de 1990 al 2001. El total de productos de software educativo analizados alcanzó cincuenta y dos, debido a que en algunas investigaciones se desarrollaron varios productos.

\section{TENDENCIA EN EL DESARROLLO DE SOFTWARE EDUCATIVO.}

Al considerar el desarrollo de software educativo como un proceso que se ha llevado a cabo durante más de una década, interesa conocer si el aporte de las universidades estatales, durante el período en cuestión, ha sido uniforme, o no. Para ello, mediante frecuencia simple, se obtuvo la Figura 1.

La Figura 1 revela, en primer lugar, que el aporte de las universidades estatales en software educativo se ha dado sin interrupciones desde 1990. Continuamente, año tras año, se han realizado investigaciones importantes en las cuales se han producido aplicaciones específicas. Los datos muestras que la tendencia es hacia un crecimiento en la cantidad de estudios efectuados por año.

También es importante reconocer que el aporte se intensificó bastante en la segunda mitad del período, partir de 1995. Cabe notar que los años 2002 y 2003 no han sido incluidos 
en el análisis, por cuanto no se dispone de la totalidad de investigaciones, al encontrarse aún varios estudios en proceso de desarrollo. Sin embargo, se conoce que la tendencia general de crecimiento se mantiene.

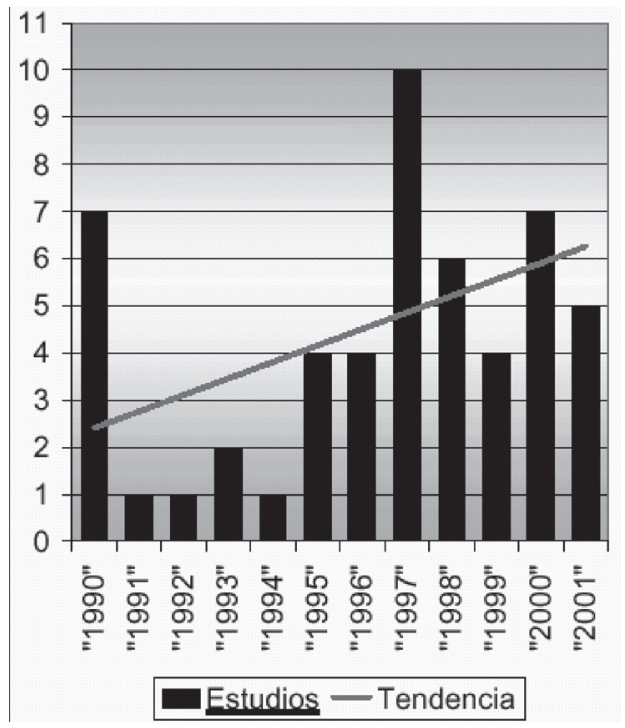

Figura 1. Investigaciones en software educativo realizadas

\section{4. ¿CÓMO SE DISTRIBUYE EL APORTE EN DESARROLLO DE SOFTWARE EDUCATIVO?}

En la Figura 2 se observa la distribución de la cantidad de estudios por universidad. El aporte dado por la Universidad de Costa Rica es similar al del Instituto Tecnológico de Costa Rica. Ambos aportes conjuntamente representan las tres cuartas partes del total de software educativo, mientras que el aporte conjunto de la Universidad Nacional y la Universidad Estatal a Distancia apenas alcanza una cuarta parte.

Cabe notar aquí dos hechos que deben ser considerados. Primero, que la Universidad Nacional comenzó su aporte a partir de 1997 mientras que la Universidad de Costa Rica conjuntamente con el Instituto Tecnológico de Costa Rica lo han mantenido durante todo el período en estudio. Segundo, que la Universidad
Estatal a Distancia no ofrece una carrera exclusivamente en el área de la Computación, como sí la ofrecen el resto de las universidades estatales.

Ambos hechos podrían ayudar a explicar los dos niveles de aporte más bajos. También se debe aclarar que varios estudios realizados por la UNED, relacionados con el Programa en Informática Educativa del MEP-FOD, no se incluyeron, dado que en estos estudios se evaluaron aspectos de dicho programa, pero no se desarrolló software educativo.

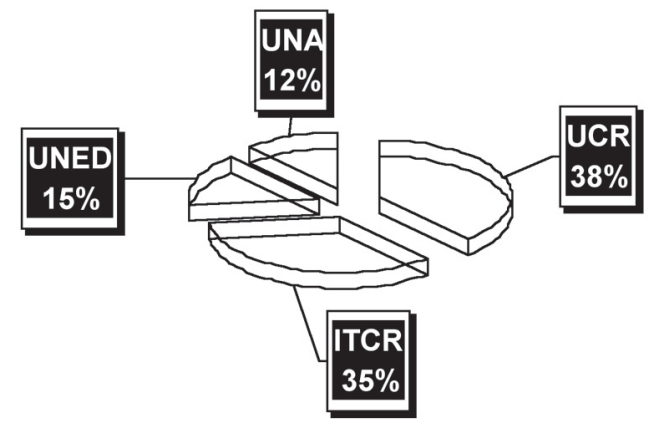

Figura 2. Aporte en Investigaciones en software educativo por universidad.

\section{UNIDADES ACADÉMICAS Y DE INVESTIGACIÓN QUE HAN APORTADO AL DESARROLLO DE SOFTWARE EDUCATIVO.}

Para efectos de este estudio, también interesa conocer cuáles unidades específicas, tales como Escuelas dentro de las Universidades Estatales, o Unidades de Investigación, han participado en el desarrollo de software educativo. La Figura 3 muestra la composición de aquellas unidades que han estado contribuyendo en el diseño y desarrollo de software educativo, dentro de las universidades estatales de Costa Rica. En esta se percibe mayor diversidad en la Universidad de Costa Rica, en cuanto a tipos de unidades que han contribuido en diseño y desarrollo de software educativo. 


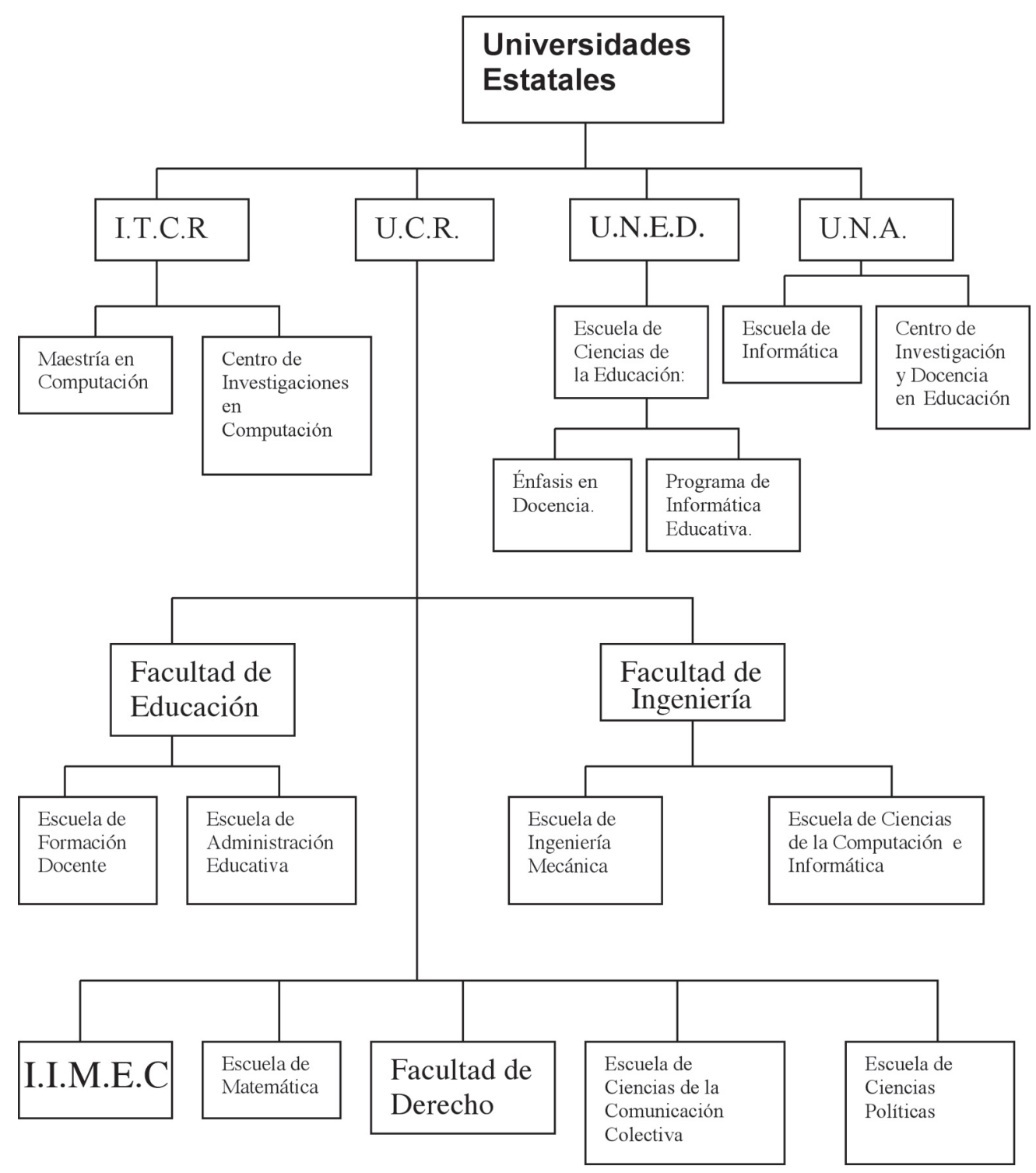

Figura 3. Unidades participantes en el diseño y desarrollo de software educativo. 
También se encontró que las unidades de computación e informática de las universidades estatales han dado el mayor aporte, seguidas por las escuelas en formación docente conjuntamente con las unidades de investigación en educación.

\section{6. ÁREAS TEMÁTICAS A LAS QUE SE HA ORIENTADO EL DESARROLLO DE SOFTWARE EDUCATIVO.}

En esta sección se muestra la totalidad de trabajos analizados en este estudio, clasificados de acuerdo con la orientación temática seguida, y por tópico dentro del área temática, lográndose así identificar aquellas áreas del conocimiento que han recibido mayor atención, así como las que han sido poco consideradas, o no consideradas del todo.

Las áreas temáticas se muestran en los cuadros del 1 al 6. Dichos cuadros también contienen información acerca de la población meta a la que se orienta el software. Posteriormente se crea la Figura 4, que resume todos los tópicos tratados con el fin de facilitar la interpretación de los datos.

Cuadro 1. Matemática

Tópico

Conocimientos básicos de probabilidad

Pruebas de bachillerato en matemática

Enseñanza de la geometría

Geometría

Álgebra elemental

Función y Ecuaciones Lineales, y Geometría Plana

Razonamiento lógico-matemático

Álgebra elemental

Conceptos básicos en Matemática

Lógica Proposicional

Geometría

Teoría de Conjuntos
Población meta

Nivel universitario, estudiantes de Ingeniería.

Estudiantes de quinto año y sus profesores de matemática y personal del Kiosco de Información del Centro Nacional de Didáctica.

Estudiantes de Secundaria.

Secundaria: III ciclo de la enseñanza media.

Estudiantes de secundaria de décimo año o inferior y profesores de matemática de secundaria.

Estudiantes del Tercer Ciclo de la Educación General

Básica costarricense

Adolescentes

Estudiantes de secundaria.

Primaria: niños con problemas de aprendizaje.

Universitaria.

Noveno año de la Educación General Básica.

Alumnos de la Enseñanza General Básica. 
Cuadro 2. Ciencias e Ingeniería.

\section{Tópico}

\section{Electroestática \\ Dinámica y Cinemática}

Procesamiento discreto de señales

\section{Hidráulica}

Teoría de Elementos Finitos

Reacciones químicas

Química Inorgánica: compuestos por combinación

Biología de la conservación

La célula

Ecología

\section{Población meta}

Secundaria, quinto año, 16 a 18 años.

Estudiantes universitarios de Ciencias de la

Computación o de Ingeniería de Sistemas.

Estudiantes universitarios de séptimo y octavo

semestre de Ingeniería Elétrica

Estudiantes universitarios de ingeniería Mecánica.

Estudiantes de Ingeniería Mecánica.

Secundaria. Estudiantes de octavo año con decuación curricular por Déficit Atencional.

Décimo y undécimo.

Público en general.

Estudiantes de secundaria.

Décimo y undécimo año.

Cuadro 3. Lenguaje

Tópico

\section{Lectoescritura}

Terapia del lenguaje

\section{Lecto-escritura}

Comunicaciones Usuales: orales, escritas y gráficas Vocabulario de la lengua de señas costarricense (LESCO)

Composición Básica.

Inglés: tema libre

Lecto-escritura

\section{Población meta}

Nivel preescolar, niños y niñas con edades entre 6 y 7 años.

Nivel preescolar, orientado a niños y niñas con edades comprendidas entre 5 y 7 años, que presentan retraso moderado en el desarrollo de su lenguaje.

Niños y niñas, en edades comprendidas entre los 10 y los 13 años, que cursan el quinto grado escolar. Ingenieros Mecánicos.

Nivel Preescolar: orientado a ayudar a los niños y las niñas sordos en el ciclo de preparatoria con edades comprendidas entre los 5 y 7 años.

Estudiantes de I Ciclo de la Enseñanza General Estudiantes de secundaria.

Niños adolescentes que sufren de parálisis cerebral. 
Cuadro 4. Investigación y docencia vía Internet

Tópico

Internet para la investigación en

Ciencias Políticas

Recorrido virtual por el Museo de los Niños

Investigaciones sociales y científicas inéditas

Educación Legal y nuevas tecnologías

Docencia e investigación en el área de la

Ingeniería Mecánica vía la Web
Población meta

Estudiantes de Ciencias Políticas.

Población infantil, estudiantes y público en general.

Todos los costarricenses usuarios de Internet en el país, y personas que radican afuera.

Estudiantes de la Facultad de Derecho de la

Universidad de Costa Rica.

Estudiantes de la Escuela de Ingeniería Mecánica.

Cuadro 5. Computadoras y programación

Tópico

Construcción de estrategias de aprendizaje

para la programación con LOGO-Micromundos

Programación de objetos basada en eventos

Aprendizaje de LOGO-Micromundos

Computadoras: conceptos básicos sobre

su funcionamiento

Windows, el procesador de palabras y la

hoja electrónica

Programación con LOGO y construcción

de hipertextos

Un lenguaje de programación icónico

orientado a objetos para IYULÚ

Lenguaje de programación para la especificación

de condiciones de estado para IYULÚ

LOGO y desarrollo del talento

Uso del lenguaje LOGO

Especificación formal de la estructura

de un Hipertexto

Desarrollo de software educativo con IYULÚ
Población meta

Estudiantes de cuarto y quinto año, cuyas edades están comprendidas entre los nueve y once años.

Niños y niñas con edades comprendidas entre 8 y 9 años.

Estudiantes de primaria.

Niños y niñas de 11 a 12 años de edad.

Estudiantes universitarios de enseñanza a distancia.

Primaria: estudiantes de quinto año.

Nivel escolar.

Nivel escolar.

Estudiantes talentosos de primaria.

Maestros encargados de laboratorio, maestros de preescolar, y niños entre 5 y 7 años.

Estudiantes de Ciencias de la Computación.

Nivel escolar. 


\section{Cuadro 6. Otras áreas}

Tópico

\author{
Música Estudiantes de Estudios Generales de la \\ Historia
}

Orientación Vocacional: exploración de carreras ofrecidas por las universidades estatales

Geografía de Costa Rica

Habilidad Cognitiva: discrimiminación entre figuras
Población meta

Universidad de Costa Rica. Estudiantes de Estudios Generales de la Universidad de Costa Rica.

Estudiantes de secundaria y primer ingreso a las universidades, cuya edad oscila entre catorce y veinte años

Secundaria: Sétimo año.

Enseñanza especial: niños con problemas de aprendizaje.

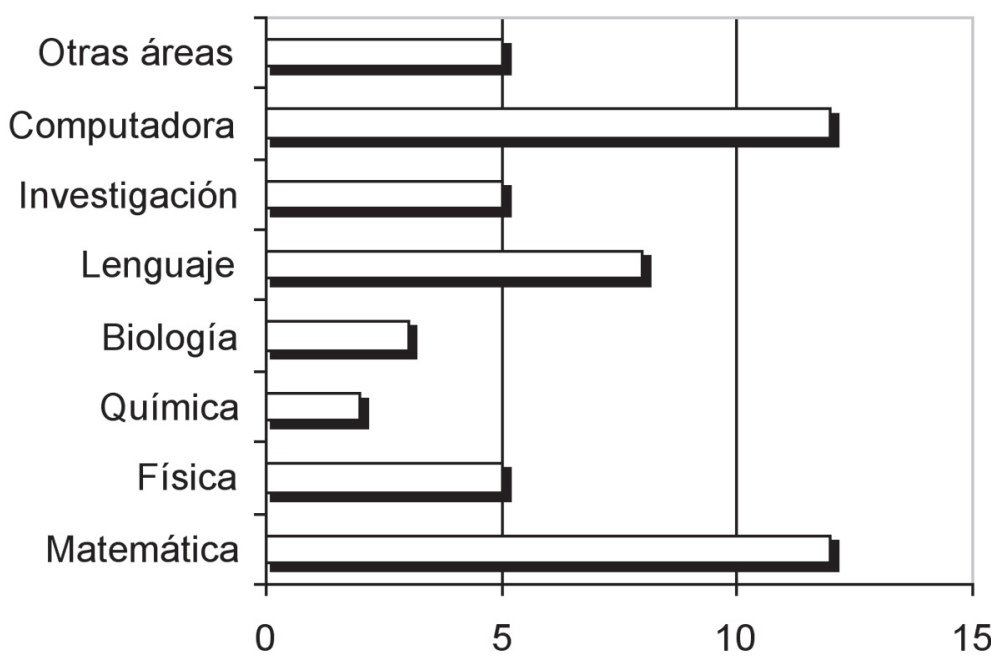

Figura 4. Áreas temáticas.

La Figura 4 muestra la distribución de las áreas de aplicación que fueron contempladas en el desarrollo de software educativo a partir de 1990. Las áreas disciplinarias que recibieron mayor atención fueron la Matemática y los estudios que tratan conceptos sobre computadoras, principalmente la programación de las mismas, seguidas por el área del Lenguaje. De manera que se ha optado por otorgarle preferencia en apoyo con software educativo a las áreas que tradicionalmente han presentado mayor grado de dificultad para los estudiantes. También se nota que a varias áreas disciplinarias se les ha brindado poca consideración, o fueron completamente desatendidas.

Respecto a la población meta a la que se orienta el software, se tiene que éste ha sido desarrollado para una amplia gama de estudiantes. Los diversos tipos de software están orientados hacia los distintos niveles de la educación formal costarricense: desde preescolar, hasta la universitaria, pero principalmente para el nivel de primaria y secundaria. Pero también hay que señalar que no todos los grados en cada nivel han sido considerados. 
Por otra parte, mientras que algunos programas están diseñados para todo tipo de público, otros se orientan a poblaciones muy particulares. En este sentido, se le ha dado importancia al desarrollo de aplicaciones para poblaciones con necesidades especiales, tales como aquellas con algún impedimento físico, o aquellas que requieren de adecuación curricular, particularmente en el área del desarrollo del lenguaje.

\section{CONCLUSIONES}

Para lograr una visión integral y profunda acerca del aporte en software con fines educativos, de más de una década, brindado por las universidades estatales costarricenses al país, en este artículo se ha analizado y presentado información valiosa al respecto, previamente no disponible.

Se considera que la información obtenida en este estudio, es valiosa en los procesos de conformación de alianzas estratégicas entre las distintas unidades organizacionales, participantes en los procesos de elaboración de software con fines educativos.

También consideramos que la información obtenida les resultará particularmente útil a todos aquellos docentes innovadores que buscan crear y utilizar software educativo para incorporarlo en su práctica educativa, ya que ahora dispondrán de información que facilita el conocer y reutilizar las experiencias previas en desarrollo de software educativo, así como el conocer adonde acudir a solicitar apoyo.

\section{BIBLIOGRAFÍA}

Bauza, G., (1997). El Guión Mutimedia. Madrid: Ediciones Anaya Multimedia, S.A.
Bermúdez, M. (1982). El análisis del contenido: procedimiento y aplicaciones. Revista Ciencias Sociales. (24) 71-80.

Cabero, J., et. al., (1999). Tecnología educativa. Madrid: Síntesis.

Galvis, A. (1994). Ingeniería de software educativo. Santa Fé de Bogotá: Ediciones UNIANDES.

Gros, B. \& Spector, M. (1994). Evaluating automated instructional design systems: a complex problem. Educational Technology, 34(5), 37-46.

Heller, R. (1991). Evaluating software: A review of the options. Computers and Education, 17(4), 285-291.

Pochet, R. (Ed.). (2000). La evaluación temática como una forma de análisis. Discurso y análisis social: métodos cualitativos y técnicas de análisis. San José: Editorial de la Universidad de Costa Rica.

\section{SOBRE EL AUTOR}

\section{Carlos Vargas Castillo}

Doctor en Educación (con distinción), Doctorado Latinomericano de la UNED, 2003.

Master of Computer Science (Becario de Fulbright-LASPAU), University of Dayton, USA. 1994.

Licenciado en Ciencias de la Computación, Universidad de Costa Rica, 1980.

Bachiller en Ciencias de la Computación, Universidad de Costa Rica, 1979.

Profesor asociado de la Universidad de Costa Rica. Escuela de Ciencias de la computación e Informática.

Teléfono: 207-4020.

Correo electrónico: cvar@costarricense.cr 
